Modern Physics Letters A,

(C) World Scientific Publishing Company

\title{
Is gravity an intrinsically quantum phenomenon? Dynamics of Gravity from the Entropy of Spacetime and the Principle of Equivalence
}

\author{
T. PADMANABHAN \\ IUCAA, P.O.Box 4, Ganeshkhind, \\ Pune 411 007, Maharashtra, India. \\ Received (received date) \\ Revised (revised date)
}

\begin{abstract}
The two surprising features of gravity are (a) the principle of equivalence and (b) the connection between gravity and thermodynamics. Using principle of equivalence and special relativity in the local inertial frame, one could obtain the insight that gravity must possess a geometrical description. I show that, using the same principle of equivalence, special relativity and quantum theory in the local Rindler frame one can obtain the Einstein-Hilbert action functional for gravity and thus the dynamics of the spacetime. This approach, which essentially involves postulating that the horizon area must be proportional to the entropy, uses the local Rindler frame as a natural extension of the local inertial frame and leads to the interpretation that the gravitational action represents the free energy of the spacetime geometry. As an aside, one also obtains a natural explanation as to: (i) why the covariant action for gravity contains second derivatives of the metric tensor and (ii) why the gravitational coupling constant is positive. The analysis suggests that gravity is intrinsically holographic and even intrinsically quantum mechanical.
\end{abstract}

\section{A possible synthesis: Motivation and summary}

Two aspects of gravity stand out among the rest as the most surprising: (i) the existence of the principle of equivalence and (ii) the connection between gravity and thermodynamics. The principle of equivalence - in the broadest sense postulates that physical phenomena taking place around any event $\mathcal{P}$ in a local region of spacetime cannot be distinguished from the corresponding phenomena taking place in a suitably chosen non inertial frame. This principle finds its natural expression when gravity is described as a manifestation of curved spacetime. The second surprise regarding gravity is the deep connection it has with thermodynamics (for a review, see references [1], [7]). Gravity is the only interaction which is capable of wrapping up regions of spacetime so that information from one region is not accessible to observers at another region. Given the fact that entropy of systems is closely related to accessibility of information, it is inevitable that there will be some connection between gravity and thermodynamics. But, in contrast to the principle of equivalence, years of research in this field (see, for a sample of references and 
related work [2]), has not led to something more profound or fundamental arising out of this feature.

It is possible to learn a lesson from the way Einstein handled the principle of equivalence and apply it in the context of the connection between thermodynamics and gravity. Einstein did not attempt to "derive" principle of equivalence in the conventional sense of the word. Rather, he accepted it as a key feature which must find expression in the way gravity is described - thereby obtaining a geometrical description of gravity. Once the geometrical interpretation of gravity is accepted, it follows that there will arise surfaces which act as one way membranes for information and thus will lead to some connection with thermodynamics. It is, therefore, more in tune with the spirit of Einstein's analysis to accept an inevitable connection between gravity and thermodynamics and ask what such a connection would imply. Let me elaborate this idea further in order to show how powerful it is.

The first step in the logic, as indicated above, is the principle of equivalence, which finds its natural expression in a model for gravity with the metric tensor of the spacetime $g_{a b}$ being the fundamental variable. This allows one to define a coordinate system around any event $\mathcal{P}$ in a region of size $L$ (with $L^{2}\left(\partial^{2} g / g\right) \ll 1$ but $L(\partial g / g)$ being arbitrary, where $\partial^{n} g$ denotes the typical value of the $n$th derivative of the metric tensor at $\mathcal{P}$ ) in which the spacetime is locally inertial. Using the laws of special relativity in this locally inertial frame and expressing them in a generally covariant manner (using the "comma-to-semicolon rule", say) one can describe the coupling of gravity to other matter fields.

As the second step, we want to give expression to the fact that there is a deep connection between one way membranes arising in a spacetime and thermodynamical entropy. This, of course, is not possible in the local inertial frame since the quantum field theory in that frame, say, does not recognize any non trivial geometry of spacetime. But it is possible to achieve our aim by using a uniformly accelerated frame around $\mathcal{P}$. In fact, around any event $\mathcal{P}$ we have fiducial observers anchored firmly in space with $\mathbf{x}=$ constant and the four-velocity $u^{i}=g_{00}^{-1 / 2}(1,0,0,0)$ and acceleration $a^{i}=u^{j} \nabla_{j} u^{i}$. This allows us to define a second natural coordinate system around any event by using the Fermi-Walker transported coordinates corresponding to these accelerated observers. I shall call this the local Rindler frame. [Operationally, this coordinate system is most easily constructed by first transforming to the locally inertial frame and then using the standard transformations between the inertial coordinates and the Rindler coordinates.] This local Rindler frame will lead to a natural notion of horizon and associated temperature. The key new idea in my approach will be to postulate that the horizon in the local Rindler frame also has an entropy per unit transverse area and demand that any description of gravity must have this feature incorporated in it.

What will such a postulate lead to? Incredibly enough, it leads to the correct Einstein-Hilbert action principle for gravity. Note that the original approach of Einstein making use of the principle of equivalence lead only up to the kinematics of gravity - viz., that gravity is described by a curved spacetime with a non trivial 
metric $g_{a b}$ - and cannot tell us how the dynamics of the spacetime is determined. Taking the next step, using the local Rindler frame and demanding that gravity must incorporate the thermodynamical aspects lead to the action functional itself.

This itself is interesting; but I will show that this approach also throws light on what has been usually considered a completely different issue: Why does the Einstein-Hilbert action contain second derivatives of the metric tensor? One must admit that this is peculiar - in the sense that no other interaction has this feature. It is also closely related to the geometrical nature of gravity which prevents the construction of a covariant scalar which is quadratic in the first derivatives of gravity. The analysis presented in this paper "builds up" the Einstein-Hilbert action from its surface behaviour and, in this sense, shows that gravity is intrinsically holographic. In the literature, the term 'holographic' is used with different meanings 4 ; I use this term with the specific meaning that given the form of the action on a two dimensional surface, there is a way of obtaining the full bulk action. In fact, this can be done for any theory but it finds a natural place only in the case of gravity.

In the $(3+1)$ formalism, this leads to the interpretation of the gravitational action as the free energy of spacetime. Einstein's equations are equivalent to the principle of minimization of free energy in thermodynamics.

This approach opens up a new point of view regarding gravity. Using principle of equivalence and special relativity in the local inertial frame, one could obtain the insight that gravity must possess a geometrical description. Using the same principle of equivalence, special relativity and quantum theory in the local Rindler frame one can obtain the dynamics of the spacetime. The key input is the connection between entropy and the horizon area and this requires introduction of a length scale into the problem which needs to be determined from observations. With hindsight, we know that this length scale will be proportional to the Planck length $L_{P}=\left(G \hbar / c^{3}\right)^{1 / 2}$. But in the approach advocated here, it is more natural to write the Newtonian gravitational force as $F=\left(c^{3} L_{P}^{2} / \hbar\right)\left(m_{1} m_{2} / r^{2}\right)$ suggesting that gravity is intrinsically quantum mechanical. The broader implications are discussed in the last section.

\section{Action functionals with second derivatives}

As described in the previous section, I will introduce a new postulate which relates the transverse spatial area of the horizon in the local Rindler frame to the entropy of the horizon. Since the entropy can be related to the Euclidean action we will be able to determine the form of the action. It turns out, however, that it is slightly easier to reach this goal if I start with some general results regarding action functionals and then connect it up with the problem at hand. This is important because some of these results do not seem to received adequate attention in the literature.

Consider a physical system described by the dynamical variables $q$ and a Lagrangian $L(\partial q, q)$ made of the dynamical variables and their first derivatives. The 
Is gravity quantum mechanical ...

ideas described below work for any number of variables (so that $q$ can be a multicomponent entity) dependent on space and time. But I shall illustrate the idea first in the context of point mechanics. Given any Lagrangian $L(\partial q, q)$ involving only up to the first derivatives of the dynamical variables, it is always possible to construct another Lagrangian $L^{\prime}\left(\partial^{2} q, \partial q, q\right)$, involving second derivatives such that it describes the same dynamics 0 . The prescription is:

$$
L^{\prime}=L-\frac{d}{d t}\left(q \frac{\partial L}{\partial \dot{q}}\right)
$$

While varying the $L^{\prime}$, one keeps the momenta $(\partial L / \partial \dot{q})$ fixed at the endpoints rather than $q^{\prime}$ s. This is most easily seen by explicit variation; we have

$$
\begin{aligned}
\delta A^{\prime} & =\int_{\mathcal{P}_{1}}^{\mathcal{P}_{2}} d t\left[\frac{\partial L}{\partial q} \delta q+\frac{\partial L}{\partial \dot{q}} \delta \dot{q}\right]-\left.\delta\left(q \frac{\partial L}{\partial \dot{q}}\right)\right|_{\mathcal{P}_{1}} ^{\mathcal{P}_{2}} \\
& =\int_{\mathcal{P}_{1}}^{\mathcal{P}_{2}} d t\left[\frac{\partial L}{\partial q}-\frac{d}{d t}\left(\frac{\partial L}{\partial \dot{q}}\right)\right] \delta q+\left.\delta q\left(\frac{\partial L}{\partial \dot{q}}\right)\right|_{\mathcal{P}_{1}} ^{\mathcal{P}_{2}}-\left.\delta q\left(\frac{\partial L}{\partial \dot{q}}\right)\right|_{\mathcal{P}_{1}} ^{\mathcal{P}_{2}}-\left.q \delta\left(\frac{\partial L}{\partial \dot{q}}\right)\right|_{\mathcal{P}_{1}} ^{\mathcal{P}_{2}} \\
& =\int_{\mathcal{P}_{1}}^{\mathcal{P}_{2}} d t\left[\frac{\partial L}{\partial q}-\frac{d}{d t}\left(\frac{\partial L}{\partial \dot{q}}\right)\right] \delta q-\left.q \delta p\right|_{\mathcal{P}_{1}} ^{\mathcal{P}_{2}}
\end{aligned}
$$

If we keep $\delta p=0$ at the end points while varying $L^{\prime}$, then we get back the same Euler-Lagrange equations as obtained by varying $L$ and keeping $\delta q=0$ at end points. Since $L=L(\dot{q}, q)$, the quantity $q(\partial L / \partial \dot{q})$ will also depend on $\dot{q}$. So the term $d(q \partial L / \partial \dot{q}) / d t$ will involve $\ddot{q}$. Thus $L^{\prime}$ contains second derivatives of $q$ while $L$ contains only up to first derivatives. In spite of the fact that $L^{\prime}$ contains second derivatives of $q$, the equations of motion arising from $L^{\prime}$ are only second order for variation with $\delta p=0$ at end points. It can be shown that, in the path integral formulation of quantum theory, the modified Lagrangian $L^{\prime}$ correctly describes the transition amplitude between states with given momenta (see p. 170 of [5]).

In the case of flat spacetime quantum field theory of a scalar field, say, we can start with a Lagrangian density of the form $L(\partial \phi, \phi)$ and obtain $L^{\prime}\left(\partial^{2} \phi, \partial \phi, \phi\right)$ by a similar procedure. In terms of the actions, the relation will be

$$
A^{\prime}=\int_{\mathcal{V}} d^{4} x L^{\prime}\left(\partial^{2} \phi, \partial \phi, \phi\right)=\int_{\mathcal{V}} d^{4} x L(\partial \phi, \phi)-\int_{\mathcal{V}} d^{4} x \partial_{a}\left[\phi \frac{\partial L}{\partial\left(\partial_{a} \phi\right)}\right] \equiv A-\mathcal{S}
$$

The second term $\mathcal{S}$ can, of course, be converted into a surface integral over the 3 -dimensional boundary $\partial \mathcal{V}$. If we consider a static field configuration (in some Lorentz frame) then the second term in (3) will have the integrand $\nabla \cdot[\phi(\partial L / \partial(\nabla \phi)]$ which can be converted to an integral over a two dimensional surface on the boundary $\partial \partial \mathcal{V}$. Taking the time integration over an interval $(0, T)$, the second term in 
(3), for static field configurations, will reduce to

$$
\mathcal{S}=\int_{0}^{T} d t \int_{\partial \mathcal{V}} d^{3} x \nabla \cdot\left[\phi \frac{\partial L}{\partial(\nabla \phi)}\right]=T \int_{\partial \partial \mathcal{V}} d^{2} x \hat{\mathbf{n}} \cdot \phi \frac{\partial L}{\partial(\nabla \phi)} \equiv \int_{\partial \partial \mathcal{V}} d^{2} x \hat{\mathbf{n}} \cdot \mathbf{P}
$$

This procedure allows one to reconstruct the bulk action if the surface term is known. As an example, let us assume the surface term has the above form with $\mathbf{P}=$ $-(T / 2) \nabla\left(\phi^{2}\right)$. This shows that $[\partial L / \partial(\nabla \phi)]=-\nabla \phi$ leading to $L=-(1 / 2)(\nabla \phi)^{2}-$ $V(\phi)$ (where $V$ is an arbitrary scalar function of $\phi$ ) for static field configurations. Lorentz invariance now allows us to fix the time dependence leading to $L=(1 / 2)\left[\partial_{a} \phi \partial^{a} \phi\right]-V$. This is the standard first order Lagrangian; adding the surface term will give the second order Lagrangian for a scalar field to be $L^{\prime}=-\phi \square \phi-(1 / 2)\left[\partial_{a} \phi \partial^{a} \phi\right]-V$.

While this procedure is viable and consistent, it is not of much use in this context and, more importantly, suffers from the following serious flaws: (i) There is no real reason or motivation to believe that the $\mathbf{P}$ in the surface term in (仼) should be of the form $-(T / 2) \nabla\left(\phi^{2}\right)$. (ii) The scaling with respect to $T$ in the postulated surface term is strange and there is no natural value for $T$. (iii) The Lagrangian $L^{\prime}$ which one obtains by this method is in no way superior to the standard Lagrangian $L$. Because of these reasons, one might as well postulate the original Lagrangian rather than obtain it in such a convoluted way.

I will now show that the situation is very different in the case of gravity and a similar procedure appears to be natural and logical at every step of the way.

\section{Gravitational dynamics from spacetime thermodynamics}

After the warm up regarding action functionals containing second derivative of dynamical variables, let us now proceed to implement the basic idea introduced in section 1. The principle of equivalence leads to a geometrical description of gravity in which $g_{a b}$ are the fundamental variables. We expect the dynamics of gravity to be described by some unknown action functional

$$
A=\int d^{4} x \sqrt{-g} L(g, \partial g) \equiv \int d^{4} x \sqrt{-g} L(g, \Gamma)
$$

involving $g_{a b} \mathrm{~s}$ and their first derivatives $\partial_{c} g_{a b}$ or, equivalently, the set $\left[g_{a b}, \Gamma_{j k}^{i}\right]$ where $\Gamma \mathrm{s}$ are the standard Christoffel symbols. From the discussion in the last section, it is obvious that the same equations of motion can be obtained from another (as yet unknown) action:

$$
\begin{aligned}
A^{\prime} & =\int d^{4} x \sqrt{-g} L-\int d^{4} x \partial_{c}\left[g_{a b} \frac{\partial \sqrt{-g} L}{\partial\left(\partial_{c} g_{a b}\right)}\right] \equiv \int d^{4} x \sqrt{-g} L-\int d^{4} x \partial_{c}\left[g_{a b} \pi^{a b c}\right] \\
& \equiv A-\int d^{4} x \partial_{c}\left(\sqrt{-g} V^{c}\right) \equiv A-\int d^{4} x \partial_{c} P^{c}
\end{aligned}
$$

where $V^{c}$ is made of $g_{a b}$ and $\Gamma_{j k}^{i}$. Further, $V^{c}$ must be linear in the $\Gamma^{\prime}$ 's since the original Lagrangian $L$ was quadratic in the first derivatives of the metric. Since $\Gamma$ s 
vanish in the local inertial frame and the metric reduces to the Lorentzian form, the action $A$ cannot be generally covariant. However, the action $A^{\prime}$ involves the second derivatives of the metric and hence we shall demand that the action $A^{\prime}$ must be generally covariant.

To obtain a quantity, which is linear in $\Gamma$ s and having a single index $c$, from $g_{a b}$ and $\Gamma_{j k}^{i}$, we must contract on two of the indices on $\Gamma$ using the metric tensor. Hence the most general choice for $V^{c}$ is the linear combination

$$
V^{c}=\left(a_{1} g^{c k} \Gamma_{k m}^{m}+a_{2} g^{i k} \Gamma_{i k}^{c}\right)
$$

where $a_{1}$ and $a_{2}$ are numerical constants. Using the identities $\Gamma_{k m}^{m}=\partial_{k}(\ln \sqrt{-g})$, $\sqrt{-g} g^{i k} \Gamma_{i k}^{c}=-\partial_{b}\left(\sqrt{-g} g^{b c}\right)$, we can rewrite the expression for $P^{c} \equiv \sqrt{-g} V^{c}$ as

$$
P^{c}=\sqrt{-g} V^{c}=c_{1} g^{c b} \partial_{b} \sqrt{-g}+c_{2} \sqrt{-g} \partial_{b} g^{b c}
$$

where $c_{1} \equiv a_{1}-a_{2}, c_{2} \equiv-a_{2}$ are two other numerical constants. If we can fix these coefficients by using a physically well motivated prescription, then we can determine the surface term and by integrating, the Lagrangian $L$. I will now show how this can be done.

Let us consider a static spacetime in which all $g_{a b}$ s are independent of $x^{0}$ and $g_{0 \alpha}=0$. Around any given event $\mathcal{P}$ one can construct a local Rindler frame with an acceleration of the observers with $\mathbf{x}=$ constant, given by $a^{i}=(0, \mathbf{a})$ and $\mathbf{a}=$ $\nabla\left(\ln \sqrt{g_{00}}\right)$. This Rindler frame will have a horizon which is a plane surface normal to the direction of acceleration and a temperature $T=|\mathbf{a}| / 2 \pi$ associated with this horizon. I shall postulate that the entropy associated with this horizon is proportional to its area or, more precisely,,

$$
\frac{d S}{d A_{\perp}}=\frac{1}{\mathcal{A}_{P}}
$$

where $\mathcal{A}_{P}$ is a fundamental constant with the dimensions of area. It represents the minimum areas required to hold unit amount of information and our postulate demands that this number be finite. Given the temperature of the horizon, one can construct a canonical ensemble with this temperature and relate the Euclidean action to the thermodynamic entropy (see, e.g, [6],[7]). Since the Euclidean action can be interpreted as the entropy in the canonical ensemble, I will demand that the quadratic action $A$ in equation (5) should be related to the entropy by $S=-A$ (with the minus sign arising from standard Euclidean continuation 6), when evaluated in the local Rindler frame with the temperature $T$. I next note that the action $A^{\prime}$ is generally covariant and hence will vanish in the local Rindler frame. It follows that the numerical value of the action $A$ in the local Rindler frame is the same as the surface term in equation (6). That is, in the static local Rindler frame, we have

$$
A=\int_{\partial \mathcal{V}} d^{4} x \partial_{c} P^{c}=\int_{0}^{\beta} d t \int_{\partial \mathcal{V}} d^{3} x \nabla \cdot \mathbf{P}=\beta \int_{\partial \partial \mathcal{V}} d^{2} x_{\perp} \hat{\mathbf{n}} \cdot \mathbf{P}
$$


I have restricted the time integration to an interval $(0, \beta)$ where $\beta=(2 \pi /|\mathbf{a}|)$ is the inverse temperature in the Rindler frame. This is needed since the Euclidean action will be periodic in the imaginary time with the period $\beta$. We shall choose the local Rindler frame such that the acceleration is along the $x^{1}=x$ axis, say, and the line element to be

$$
d s^{2}=(1+a x)^{2} d t^{2}-d \mathbf{x}^{2}
$$

Evaluating $P^{a}$ using equation (8) we find that only the $c_{1}$ term contributes and $P^{a}=\left(0,-c_{1} a, 0,0\right)$ so that the action in (10) becomes (on using $a \beta=2 \pi$ )

$$
A=-c_{1} a \beta \int d^{2} x_{\perp}=-2 \pi c_{1} \int d^{2} x_{\perp}=-2 \pi c_{1} A_{\perp}
$$

where $A_{\perp}$ is the transverse area of the $\left(x^{2}-x^{3}\right)$ plane. The entropy $S$, which is equal the Euclidean action, will be given by $S=-A$ with the minus sign arising from standard Euclidean continuation. Invoking our postulate (9) we find that $c_{1}=\left(2 \pi \mathcal{A}_{P}\right)^{-1}$.

To fix $c_{2}$, we shall repeat the same analysis in a different coordinate system representing the local Rindler frame in which the line element is given by

$$
d s^{2}=(1+2 a x) d t^{2}-\frac{d x^{2}}{(1+2 a x)}-d x_{\perp}^{2}
$$

In this case, only the $c_{2}$ term in (8) contributes giving again $P^{a}=\left(0,-2 a c_{2}, 0,0\right)$. The action now becomes

$$
A=-2 a c_{2} \beta \int d^{2} x_{\perp}=-4 \pi c_{2} \int d^{2} x_{\perp}=-4 \pi c_{2} A_{\perp}
$$

By the same argument as before, we get $c_{2}=\left(4 \pi \mathcal{A}_{P}\right)^{-1}$. Hence $P^{c}$ has the form

$$
P^{c}=\frac{1}{4 \pi \mathcal{A}_{P}}\left(2 g^{c b} \partial_{b} \sqrt{-g}+\sqrt{-g} \partial_{b} g^{b c}\right)=\frac{\sqrt{-g}}{4 \pi \mathcal{A}_{P}}\left(g^{c k} \Gamma_{k m}^{m}-g^{i k} \Gamma_{i k}^{c}\right)
$$

[The second equality is obtained by using the standard identities mentioned after equation (7).] This result is remarkable and let me discuss it before proceeding further.

The general form of $P^{c}$ which we obtained in (8) is not of any use unless we can fix $\left(c_{1}, c_{2}\right)$. For static configurations, we can convert the extra term to an integral over time and a two-dimensional spatial surface. This is in general true, as we saw in the case of the scalar field earlier in section 2. But in general, the result will not have any simple form and will involve an undetermined range of integration over time coordinate (like the factor $T$ we found in the case of the scalar field). But in the case of gravity, three natural features conspire together to give an elegant form to this surface term. First is the fact that Rindler frame has a periodicity in Euclidean time and the range of integration over the time coordinate is naturally restricted to the interval $(0, \beta)=(0,2 \pi / a)$. The second is the fact that the integrand $P^{c}$ is 
Is gravity quantum mechanical ...

linear in the acceleration $a$ thereby neatly canceling with the $(1 / a)$ factor arising from time integration. Finally, there are two natural coordinate systems for the Rindler spacetime which allows us to determine the constants $c_{1}$ and $c_{2}$. It will be incredible if all these are accidental and are not of any fundamental significance. (To be absolutely rigorous, we should verify that the form of $P^{c}$ determined in (15) will correctly reproduce the entropy as $\left(A_{\perp} / \mathcal{A}_{P}\right)$ in any other coordinate system. One can show by a fairly straightforward analysis that this is indeed true in any other static coordinates in the Rindler frame with the periodicity in Euclidean time.)

Given the form of $P^{c}$ we need to solve the equation

$$
\left(\frac{\partial \sqrt{-g} L}{\partial g_{a b, c}} g_{a b}\right)=P^{c}=\frac{1}{4 \pi \mathcal{A}_{P}}\left(2 g^{c b} \partial_{b} \sqrt{-g}+\sqrt{-g} \partial_{b} g^{c b}\right)
$$

to obtain the first order Lagrangian density. This is straightforward and we get

$$
\sqrt{-g} L \equiv \frac{1}{4 \pi \mathcal{A}_{P}}(\sqrt{-g} \mathcal{G})=\frac{1}{4 \pi \mathcal{A}_{P}}\left(\sqrt{-g} g^{i k}\left(\Gamma_{i \ell}^{m} \Gamma_{k m}^{\ell}-\Gamma_{i k}^{\ell} \Gamma_{\ell m}^{m}\right)\right)
$$

(It is easier to verify the result by calculating the derivative of (17) with respect to $g_{a b, c}$ and comparing with (16) written in terms of Christoffel symbols.) This is the second surprise. The Lagrangian which we have obtained is precisely the first order Dirac-Schrodinger Lagrangian for gravity (usually called the $\Gamma-\Gamma$ Lagrangian). Note that we have obtained it without introducing the curvature tensor anywhere in the picture. Once again, this is unlikely to be a mere accident.

Given the two pieces, the final second order Lagrangian follows from our equation (6) and, of course, it is the standard Einstein-Hilbert Lagrangian.

$$
\sqrt{-g} L_{\text {grav }}=\sqrt{-g} L-\frac{\partial}{\partial x^{c}}\left(g_{a b} \pi^{a b c}\right)=\left(\frac{1}{4 \pi \mathcal{A}_{P}}\right) R \sqrt{-g} .
$$

Thus our full second order Lagrangian turns out to be the standard Einstein-Hilbert Lagrangian. The surface terms dictate the form of the Einstein Lagrangian in the bulk. That is, the postulate of entropy being proportional to the area of the horizon, added to the requirement of general covariance, uniquely determines the gravitational action principle. The idea that surface areas encode bits of information per quantum of area allows one to determine the nature of gravitational interaction on the bulk, which is an interesting realization of the holographic principle. I will conclude this section with a set of technical comments and discuss the implications of this result in the next section.

(i) Since $\pi^{a b c}$ only depends on the derivative of the Lagrangian with respect to $g_{a b, c}$, it does not change if a scalar function of $g_{a b}$ is added to $L$ or to $\mathcal{G}$ in equation (17). Any such invariant scalar, built purely from $g_{a b}$, must be a constant. This implies that we can add to $R$ an undetermined constant in (18); thus a cosmological constant is still allowed and - unfortunately — this approach cannot say anything about cosmological constant. Also note that our approach provides, even classically, a new route to Einstein's theory. In (7), one can pull out one overall constant and 
keep the ratio $\lambda \equiv\left(a_{1} / a_{2}\right)$ as unknown. Integrating (16) and adding to the surface term will now lead to a messy expression which can be expressed as a linear combination of $R$ and a non-covariant term multiplied by $(1+\lambda)$. The vanishing of the non-covariant term will require $\lambda \equiv\left(a_{1} / a_{2}\right)=-1$ and $a_{2}$ will remain undetermined. This, while pedagogically interesting, does not lead to anything new.

(ii) The approach leads to new insights in the $(3+1)$ formalism of gravity as well. If we foliate the spacetime by a series of spacelike hyper-surfaces $\mathcal{S}$ with $u^{i}$ as normal, then $g^{i k}=h^{i k}+u^{i} u^{k}$ where $h^{i k}$ is the induced metric on $\mathcal{S}$. Given the covariant derivative $\nabla_{i} u_{j}$ of the normals to $\mathcal{S}$, one can construct only three vectors $\left(u^{j} \nabla_{j} u^{i}, u^{j} \nabla^{i} u_{j}, u^{i} \nabla^{j} u_{j}\right)$ which are linear in covariant derivative operator. The first one is the acceleration $a^{i}=u^{j} \nabla_{j} u^{i}$; the second identically vanishes since $u^{j}$ has unit norm; the third, $u^{i} K$, is proportional to the trace of the extrinsic curvature $K=\nabla^{j} u_{j}$ of $\mathcal{S}$. Thus $V^{i}$ in the surface term must be a linear combination of $u^{i} K$ and $a^{i}$ and the corresponding term in the action must have the form

$$
A_{\text {surface }}=\int d^{4} x \sqrt{-g} \nabla_{i}\left[\lambda_{1} K u^{i}+\lambda_{2} a^{i}\right]
$$

where $\lambda_{1}$ and $\lambda_{2}$ are numerical constants. (This result is known in the conventional $(3+1)$ formalism; see e.g., equation (21.88) of the first reference in [8] ). Since $u^{i} a_{i}=0$, the spacelike boundaries at $x^{0}=$ constant gets contribution only from $K$ while the time like surfaces like $x^{1}=$ constant gets contribution from the normal component of the acceleration $a^{i} \hat{n}_{i}$ where $\hat{n}_{i}$ is the normal to the time like surface. For static spacetimes with a horizon, the time integration can be limited to the range $(0, \beta)$ and $\nabla_{i} a^{i}$ becomes $\nabla_{\alpha} a^{\alpha}$. So the second term can be converted into an integral of the normal component of the acceleration $a^{\alpha} \hat{n}_{\alpha}$ over a two surface. Using $\left(a^{\alpha} \hat{n}_{\alpha}\right) \beta=2 \pi$, we find that this term is proportional to the transverse area and gives the entropy of the spacetime. Proceeding as before, one would like to obtain a first order Lagrangian whose derivative with respect to the dynamical variables will contribute to the surface term in (19). In the $(3+1)$ formalism, the spatial components of the metric are the natural dynamical variables. Requiring that the first term $\lambda_{1} K u^{i}$ in (19) arises through our prescription will lead to the following first order action

$$
A_{I}=\int d^{4} x \sqrt{-g} L=\int d^{4} x \sqrt{-g}\left[\alpha_{1} K^{\mu \nu} K_{\mu \nu}+\alpha_{2} K^{2}+F\left({ }^{3} g\right)\right]
$$

where $\alpha_{1,2}$ are numerical constants with $\left(\alpha_{1}+3 \alpha_{2}\right)=\lambda_{1}$ and $F\left({ }^{3} g\right)$ is a function of the 3-geometry. Adding (20) and (19) will give the full bulk action with three undetermined numerical constants and one unknown function $F$. Demanding general covariance for this expression will allow us, after fairly detailed algebra, to determine $F$ to be the scalar curvature ${ }^{3} R$ of the 3 -geometry and the relative values of the numerical constants. This will lead to the standard ADM form of the action functional. In the Euclidean sector, the ADM Lagrangian becomes the Hamiltonian because of the standard sign change in terms quadratic in $K^{\alpha \beta}$. The full Euclidean 
action can be written in a suggestive form as

$$
A_{\text {grav }}=A_{\text {surface }}+A_{I}=S-\beta H
$$

for any spacetime geometry having a periodicity $\beta$ in the Euclidean time. In this spirit, gravitational action represents the free energy of the spacetime; the first order term gives the Hamiltonian (in the Euclidean sector) and the surface term gives the entropy.

(iii) It is actually possible to motivate the form of $(7)$ directly from the analysis in the local Rindler frame. To see this note that (10) along with (9) requires the spatial components $V^{\alpha}$ to be proportional to the acceleration $a^{\alpha}=\Gamma_{i k}^{\alpha} u^{i} u^{k}$. Writing, $a^{\alpha}=\Gamma_{i k}^{\alpha}\left(g^{i k}-h^{i k}\right)$ the first term leads directly to the second term in (7) while an analysis of the second term in the local Rindler frame will give raise to the first term of $(7)$.

\section{Conclusions}

Let us take stock of the new features in this particular approach and see where it leads further. To begin with, we recall that this approach is a natural extension of the original philosophy of Einstein; viz., to use non inertial frames judiciously to understand the behaviour of gravity. In the original approach, Einstein used the principle of equivalence which leads naturally to the description of gravity in terms of the metric tensor. Unfortunately, classical principle of equivalence cannot take us any further since it does not encode information about the curvature of spacetime. However, the true world is quantum mechanical and one would like to pursue the analogy between non inertial frames and gravitational field into the quantum domain. Here the local Rindler frame arises as the natural extension of the local inertial frame and the study of the thermodynamics of the horizon shows a way of combining special relativity, quantum theory and physics in the non inertial frame. I have shown that these components are adequate to determine the action functional for gravity and, in fact, leads very naturally to the Einstein-Hilbert action.

All along the analysis, one cannot but notice the natural manner in which different factors blend to give meaningful results. I started with a prescription for describing any theory with first order Lagrangian in terms of another Lagrangian with second derivative terms. This prescription is quite general but it is pretty much useless in all cases except in gravity ! We saw that the Lagrangian for a scalar field, say, obtained by this prescription is in no way preferable to the standard quadratic Lagrangian for the scalar field. But in the case of gravity, the second order Lagrangian turns out to be the Einstein-Hilbert Lagrangian. This is remarkable because we did not introduce the curvature of spacetime explicitly into the discussion and - in fact - the analysis was done in a local Rindler frame which is just flat spacetime. The idea works because the action for gravity splits up into two natural parts neither of which is generally covariant. Though the sum of the 
two parts (which is generally covariant) is zero, the expression for individual parts can be ascertained in the local Rindler frame specifically because these parts are not generally covariant.

The analysis determines the action for gravity to within an undetermined multiplicative factor which we have called $\mathcal{A}_{P}$. In equations (12), (14) the product a $\beta$ has the value $2 \pi(c / \hbar)$ in normal units if the temperature is measured in energy units. Hence the two constants we determined will scale as $c_{1}=\left(\hbar / 2 \pi c \mathcal{A}_{P}\right), c_{2}=$ $\left(\hbar / 4 \pi c \mathcal{A}_{P}\right)$. The gravitational interactions are therefore determined by the coupling constant $c \mathcal{A}_{P} / \hbar$. While the existence of $c$ in the gravitational Lagrangian is completely understandable and arises from the metric having, say, a $c^{2} d t^{2}$ term, the existence of $\hbar$ in gravity requires further thought. One could, of course, trivially redefine $\mathcal{A}_{P}$ as something like $\kappa \hbar$ cancel out $\hbar$ and use $\kappa$ as a purely classical coupling constant. This is, of course, what was done historically. But it is not clear whether it hides the deeper meaning of spacetime structure. In the current approach, $\mathcal{A}_{P}$ arises as a fundamental unit of area and the coupling constant for gravity does involve $\hbar$ if $\mathcal{A}_{P}$ is taken as a basic constant. Keeping $\mathcal{A}_{P}$ independent of $\hbar$ has non trivial implications when one takes the limits $\hbar \rightarrow(0, \infty)$ and this issue needs to be analyzed further.

The fundamental postulate we use is in equation (9) and it does not refer to any horizon. To see how this comes about, consider any spatial plane, say the $y-z$ plane, in flat spacetime. It is always possible to find a Rindler frame in the flat spacetime such that the chosen surface acts as the horizon for some Rindler observer. In this sense, any plane in flat spacetime must have an entropy per unit area. Microscopically, I would expect this to arise because of the entanglement over length scales of the order of $\sqrt{\mathcal{A}_{P}}$. We have defined in $(9)$ the entropy per unit area rather than the total entropy in order to avoid having to deal with global nature of the surfaces (whether the surface is compact, non compact etc.).

An argument is sometimes advanced that Rindler (or de Sitter) horizon is conceptually different from, say, black hole horizon because the former is observer dependent. I believe this argument is incorrect and that all horizons (including even Rindler horizon) should be treated on par because of at least three reasons. (i) To begin with, if the notion of entropy in black hole spacetimes is not accidental, then one would expect any one-way-membrane which blocks out information to lead to a notion of entropy. (ii) As regards observer dependence, even in the case of Schwarzschild spacetimes, it is possible to have observers moving in time-like trajectories inside the event horizon who will access part of the information which is not available to the outside observer. It seems unlikely that these suicidal observers will attribute the same amount of entropy to the Schwarzschild black hole as an observer playing it safe by staying far away from the event horizon. (iii) If the notion of entropy associated with a one way membrane arises from local degrees of freedom and Planck scale physics (as in the case of entanglement entropy) then it should be a local construct. This is also in consonance with the spirit that all physical phenomena must be local and the fact that principle of equivalence operates in a 
local region.

This approach also provides a natural explanation as to why the gravitational coupling constant is positive. It is positive because entropy and area are positive quantities.

This approach emphasizes the role of two dimensional surfaces in fundamental physics once again, which was noted earlier in the world sheet action for strings and in the quantization of areas in loop gravity $\mathrm{6}$. A two dimensional surface is the basic minimum one needs to produce region of inaccessibility and thus entropy from lack of information. When one connects up gravity with spacetime entropy it is is inevitable that the coupling constant for gravity has the dimensions of area in natural units. The next step in such an approach will be to find the fundamental units by which spacetime areas are made of and provide a theoretical, quantum mechanical description for the same. This will lead to the proper quantum description of spacetime with Einstein action playing the role of the free energy in the thermodynamic limit of the spacetime.

I thank Apoorva Patel, K.Subramanian and J.V.Narlikar for comments on the manuscript.

\section{References}

1. Birrell N.D and Davies P.C.W, Quantum fields in curved space, (Cambridge University Press, Cambridge, 1982);

2. Bekenstein, J.D., Phys. Rev. D 7, 2333 (1973); Hawking S.W., Comm.Math.Phys., 43, 199 (1975) Gerlach, U.H., Phys. Rev. D 15, 1479 (1976); t'Hooft, G., Nucl. Phys. B256, 727 (1985). York, J., Phys. Rev. D 15, 2929 (1985); Zurek, W.H. and Thorne, K.S., Phys. Rev. lett. 54, 2171 (1985); Bombelli. L et al., , Phys. Rev. D 34, 3, 73 (1986). For an earlier attempt, similar in spirit to the current paper, see Jacobsen, T. Phys.Rev.Letts., 75, 1260 (1995).

3. This idea was suggested in T. Padmanabhan, The Holography of gravity encoded in a relation between Entropy, Horizon Area and the Action for gravity [Second Prize essay; Gravity Research Foundation Essay Contest, 2002]; the current paper elaborates on this idea.

4. 't Hooft, G Dimensional Reduction in quantum gravity, gr-qc/9310026: The holographic principle, hep-th/0003004; L. Susskind (1995) J.Math.Phys., 36, 6377; for a recent review, see R. Bousso,, The holographic principle, hep-th/0203101.

5. D. Lynden-Bell and T. Padmanabhan, unpublished; Padmanabhan,T., Cosmology and astrophysics - through problems (Cambridge university press, 1996) p. 170; p. 325.

6. Padmanabhan T., gr-qc/0204019 (2002); gr-qc/020208d (2002).

7. Padmanabhan T., gr-qc/0202078, Mod. Phys. Letts.A, (2002) (to appear).

8. Miser, C.W., Thorne K.S., Wheeler, J.A. , Gravitation (Freeman and co., 1973), p.520; York,J.W (1987) in Between Quantum and Cosmos, eds W.H.Zurek et al (Princeton University Press, Princeton, 1988), p.246.

9. Ashtekar A Rovelli C and Smolin L 1992 Phys Rev Letts 69237. 